\title{
CONF-841117--33
}

\section{RADIOLOGICAL SAFETY AT ARGONNE NATIONAL LABORATORY'S HEAVY ION RESEARCH FACILITY}

R. H. Cooke and R. A. Wynveen, Argonne National Laboratory

$\operatorname{CONF}-841117--33$

Abstract DE85 005027

This paper discusses the radiological safety system to be employed at the Argonne Tandem-LINAC Accelerator system (ATLAS). The design parameters of ATLAS that affect safety have remained unchanged since ATLAS construction began in 1982. The specialized radiological safety considerations of ATLAS were discussed in 1982 (1). This paper will present the details of the hardware, the administrative controls, and the radiation monitoring that will be in effect when beam is produced in April 1985. The experimental hall utilizing the maximum energy beam ( $\sim 27 \mathrm{MeV}$ per nucleon) from the completed ATLAS has been partitioned with shielding blocks into its final configuration. Because scientists want access to some of the partitioned-off areas while beam is present in other areas, an interlock and logic system allowing such occupancy has been designed. The rationale and hardware of the system will be discussed. Since one of the potential radiation hazards is high-energy forward-directed neutrons from any location where the beam impinges (such as collimators, bending and focussing systems, experimental targets, and beam stops), radiation surveys and hazard assessments are necessary for the administrative controls that allow occupancy of various areas. Because of the various uses of ATLAS, neutrons (the dominant beam hazard) will be non-existant in some experiments, will be of energies $\gtrsim 10 \mathrm{MeV}$ for a few experiments. These conditions may exist at specific locations during beam preparation but may change rapidly when beam is finally 
delivered to an experimeatal area. Monitoring and assessing such time varying and geographically changing hazards will be a challenge since little data will be available on source terms until various beams are produced of sufficient intensity and energy to make measurements. How the operating division for ATLAS and the Argonne safety division are addressing this aspect through administrative controls will also be discussed.

\section{Introduction}

The Argonne Tandem-LINAC Accelerator System (ATLAS) at Argonne National Laboratory (ANL) is a user-oriented national facility for nuclear physics research. A discussion of some specialized radiological safety considerations unique to the facility was presented in 1982 (1).

Figure 1 shows the ATLAS Facility. The presently existing booster LINAC, consisting of 24 independently-phased superconducting split-ring resonators that are housed in four cryostats, delivers beam into Target Area II. An additional 18 resonators, housed in three newly constructed cryostats, are now being fabricated and installed in a new beam tunnel. This will permit beams with energies up to $27 \mathrm{MeV}$ per nucleon and ions with up to 120 nucleons to be delivered to Target Area III. The final configuration of shielding walls in Target Area III is shown in Figure 1.

This paper discusses the safety system that will be employed for the facility when it becomes operational, emphasizing the safety system to be employed within Target Area III. 


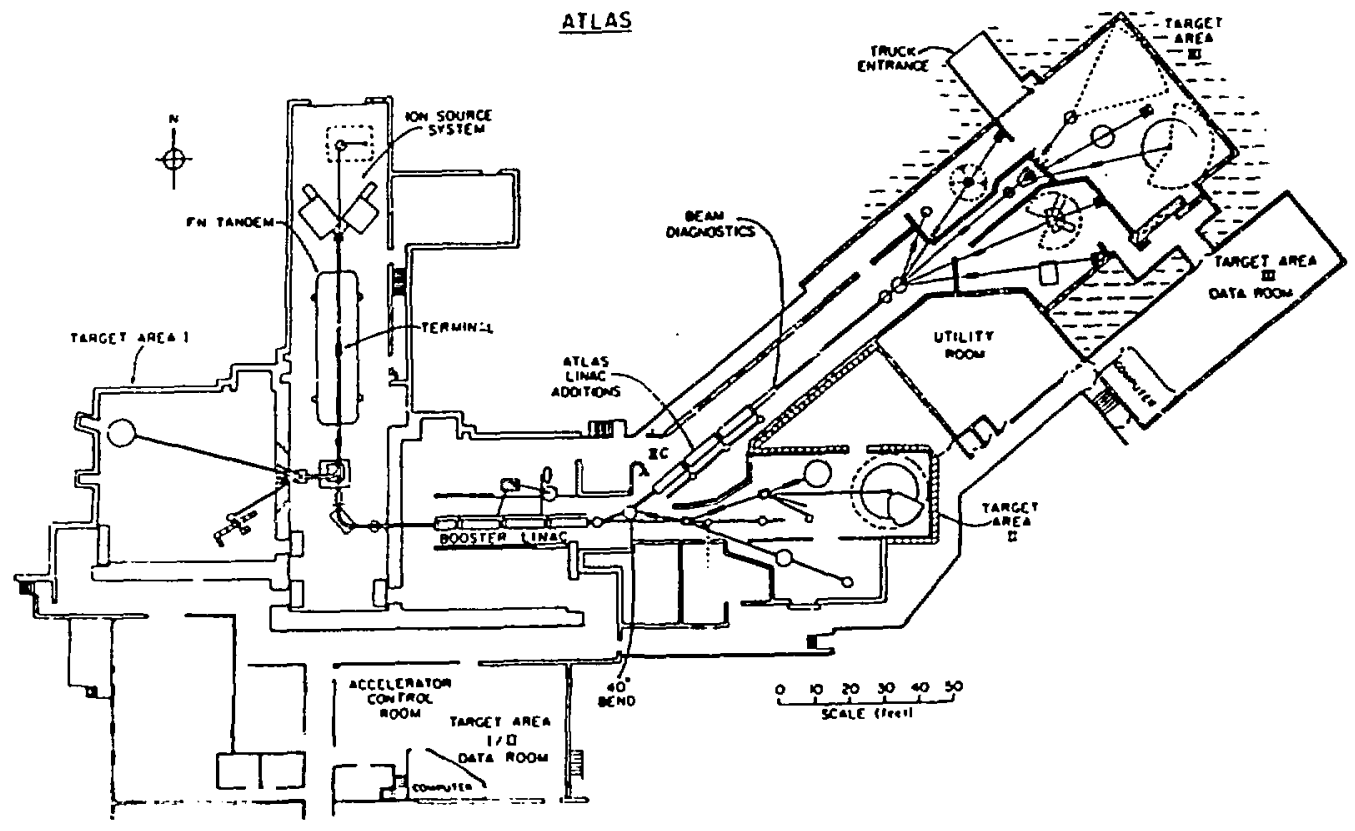

Figure 1. Plan View of ATLAS

\section{Current and Expected Radiological Hazards}

The radiolugical safety hazards previously described (1) continlie to exist. In addition, a neutron hazard has been observed within the vault and at any point downstream of the high-energy end of the tandem when light or loosely-bound ions are accelerated. On the rare occasions that such beams are accelerated, the vault and booster LINAC rooms are not occupied during tuning and focussing. Occupancy during normal operation is permitted only after a radiation survey has been performed. As projectile energies increase, higher energy $(>10 \mathrm{MeV})$ forward-directed neutrons are expected from any beam species. However, for the 
maximum energies currently available from the booster LINAC (approximately $11 \mathrm{MeV}$ per nucleon), only isotropic evaporation neutrons have been noted in Target Area II.

The previously projected radiation hazards for the new facility along with the operating experience with the current facility form the design basis of the new portion of the safety system. Both higher evaporation neutron and gamma flux densities are expected at all locations from the $40^{\circ}$ bending system, downstream through the cryostats into Target Area III. The high energy forward-directed neutrons, in addition to evaporation neutrons, are also expected at all points where beam energies exceed $\sim 11 \mathrm{MeV} / \mathrm{A}$ or some greater energy as determined by later measurements. It is expected that access to areas other than where beam is delivered and stopped will be routinely desired by experimenters.

\section{Radiation Safety System and Hazard Control}

A. Currently Operating Facility - The existing safety system consists of a combination of hardware and administrative controls that are supported by radiation surveys. The specification of a safety system is the responsibility of the Physics Division which operates ATLAS. However, the system is determined in consultation with the Health Physics Section of the Occupational Health and Safety Department, an administratively separate group. The hardware is maintained by the operating division but tested by the safety group.

Similarly, the administrative controls are auministered by the operating division with surveys and safety interlock bypass approvals obtained from the safety 
group. The safety system is designed to allow experimenters maximum access while controlling radiation exposures to levels consistent with the ALARA philosophy. A measure of the success of the current approach is that there have been few positive exposures noted by the neutron and gamma personnel dosimeters.

The administrative controls include criteria under which various interlocks and hardware can be bypassed to allow personnel access to various areas. The bypass authorizations are based on actual radiation measurements under certain machine conditions. Access is allowed under two different circumstances. When conditions are not likely to produce radiation fields of concern, access is allowed immediately with the requirement that a radiation survey must be performed as soon as possible to verify the safe condition. When conditions are very likely to produce radiation fields of concern or when the likelihood is unknown, no access is allowed until a radiation survey is performed to confirm that access is safe. Details of the present radiation safety system and administrative controls are provided in the previously indicated reference.

B. New Facility - When beam is delivered to Target Area III beginning approximately April 1985, the exf : led safety system and adininistrative controls will embody the approach currently employed. An iterative approach to implementing the safety system is expected to allow maximum access consistent with a highlevel of safety while information is acquired about the hazards that exist within the various areas of the new facility. This planned approach is seemingly novel in that it does not specify all of the safety-related controls in 
advance of the actual operation. It is felt that this is the most costeffective course of action.

The envisioned hardware includes interlocked gates for each experimental area, visible and audible indicators, and scram switches. Figure 2 shows the locations for the various pieces of hardware in Target Area III. The openings which are designated with an "I" are interlocked to exclude beam from the entire target area when any activated interlock is interrupted. All of the interior openings will have interlocked gates. The pedestrian and outside service entrances at the top of Figure 2 will have interlocked doors, and the entrance to the labyrinth at the bottom of Figure 2 will have an interlocked shielding door.

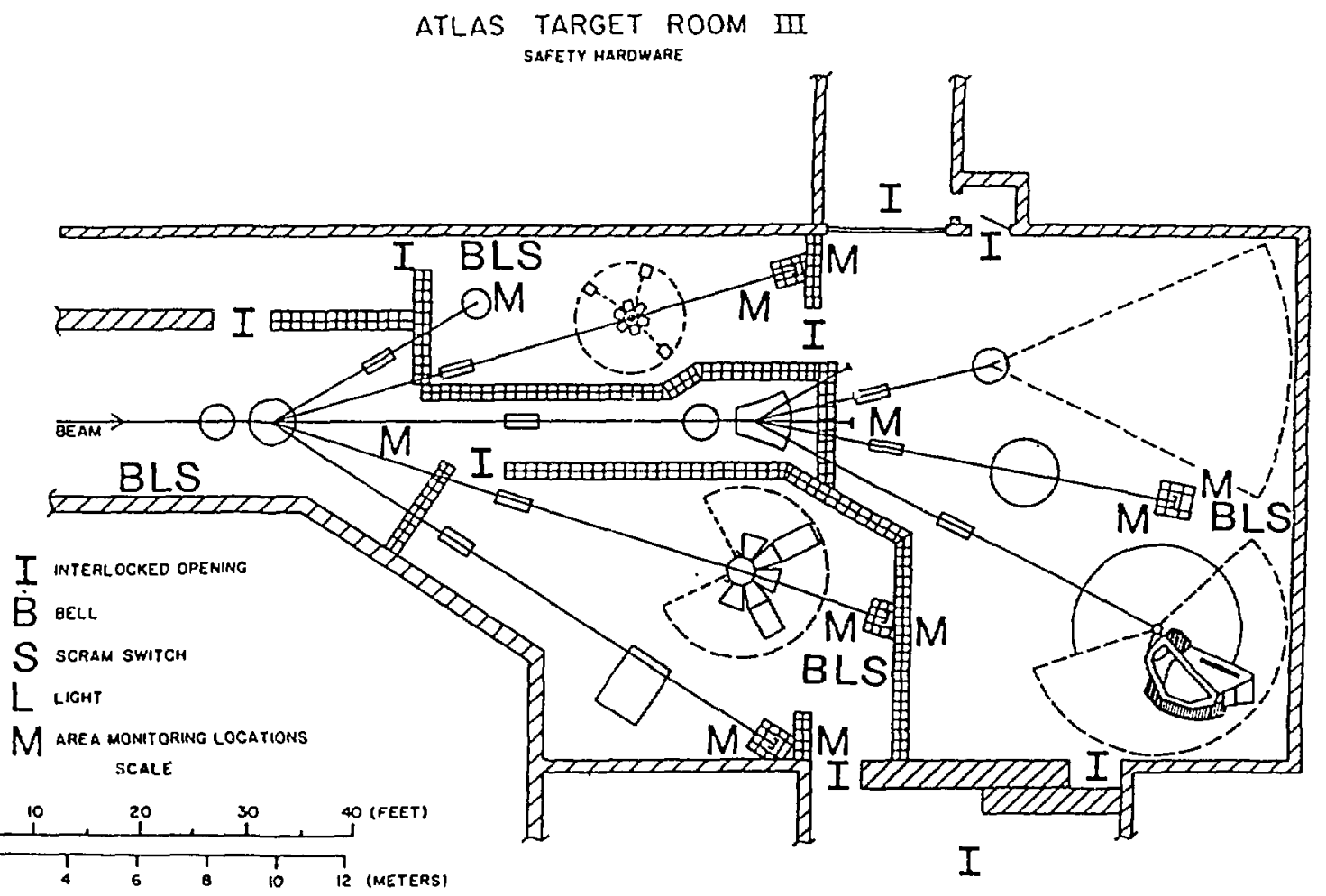

Figure 2. Locations of Safety Hardware in Target Area I1I. 
In order to deliver beam to an area, a reset switch within the experimental area will have to be set and all the interlocks for that area made good within a given period of time. A second reset switch at the main control panel will have to be set next. If an interlock is interrupted after the local reset switch has been set and after the interlocks have been made good, then the entire process will have to be repeated before beam can be delivered to the area. This procedure has been established hecause the facility is so large with many pathways between two locations.

There will be scram switches in each of the experimental areas of Target Area III as depicted in Figure 2. When the scram is activated, beam will not be accelerated into Target Area III. The scram switches will have to be reset locally before beam can be reintroduced into the area.

There will be audible (bell) and visual (light) indicators in each of the experimental areas as shown in Figure 2. When beam is to be introduced into an area, the audible and visual indicators will be activated approximately 15 seconds prior to the beam introduction.

There will be area monitors within each occupiable. Just how to conduct the area monitoring and just where to place area monitors when one has such time varying, geographically changing and energy dependent hazards, are questions that can best be answered when Target Area IlI is actually utilized.

All or any combination of the interlocks can be bypassed to allow access if the personnel exposure potential is known to be acceptable. The bypass can be 
activated by a key that will be in the possession of the ATLAS operator. Initially, there will be no bypasses permitting access to Target Area III while beam tuning and focussing operations are taking place. During normal running, bypasses will initially be based on the same criteria as presently used for Target Area II. All bypasses will be backed up by radiation surveys of the area.

When there is a possibility of high-energy forward-directed neutrons, one must be able to identify such neutrons, then determine the hazard to personnel before authorizing a bypass. A movable instrument may be used to quantify the flux density, eqergy, and thus the hazard from a given neutron field for a given set of conditfion. Since the neutrons can be produced at any number of locations and sincle the orward-directed neutrons will be present within a fairly small solid angle, onf needs a truly portable instrument to make measurements at various locations in the area of interest. The instrument must have energy response characteristics that are appropriate for the expected neutron energies. More than one instrument may even be required. A hazard evaluation must be made in a timely manner and without complication in order for personnel to have access to desired areas. The area monitors must provide a reasonable back-up to the portable instrument radiation survey so that personmel in the area will be alerted to any increased hazard resulting from changed beam condition. Until the facility becomes operational and measurements can be made, no instruments will be specified and, therefore, access will not be permitted for those operating conditions where the use of a rem meter is inappropriate. 
Ultimately, the objective is to produce a catalogue of hazard conditions or an algorithm that will accurately predict the potential for a radiation hazard. These will be used by a controlling computer that will require interlocks to be active before hazardous beamcan be be delivered to an occupiable area. These interlocks will only be bypassed when appropriate survey instruments and area monitors are available and operational.

\section{Radiation Measurements}

Both gamma and neutron measurements will be required in both Target Areas II and III as an administrative control procedure. The gamma measurements are expected to be straight forward using currently available hand-held instruments. However, when neutrons are present, they rapidly become the dominant hazard and it becomes more difficult to interpret the measurement results in terms of the potential radiation exposure hazard.

Neutron measurements are made in Target Area II using a Bonner Sphere Spectrometry System. The Bonner Sphere detectors and the necessary computer code have been cross-checked with NBS for neutron flux densities and dose equivalent rates. The dose equivalent rates obtained from the Bonner Sphere system are in good agreement with the NBS values. The energy spectrum provided by this system includes thermal neutrons through $100 \mathrm{MeV}$ neutrons. Little detail is provided in the energy range from 1 to $20 \mathrm{MeV}$. The Bonner Sphere system is cumbersome to use and does not lend itself to providing hazard information in a short period of time. A fairly steady state flux of neutrons must be present in order to obtain good results. It, therefore, has limited application for use as a routine monitoring instrument. 
A portable rem meter, consisting of a boron triflouride tube inside of a 10-inch spherical moderator is currently used to measure neutrons in Target Area II. As is the case with most rem meters employing a moderator, it provides a reasonably accurate readout in mrem/h for neutron energies up to, at most, about 10 MeV. Since no fast forward-directed neutrons have been seen with the Bonner Sphere system at the energies and currents available in Target Area II, this type of portable neutron instrument is adequate for area surveys in Target Area II. Similarly constructed permanent area neutron monitors have been installed in Target Area II and in areas upstream of Target Area II.

From the measurements made to date in Target Area II, it appears that there is a possibility of developing an algorithm for predicting the neutron hazard from evaporation neutrons. The algorithm is based on machine parameters such as ion species, beam current, and beam energy in MeV/nucleon. It has also become apparent that a spectrometry system is needed which is capable of seeing details in the energy spectrum above $1 \mathrm{MeV}$. This would differentiate between evaporation neutrons and any fast foward-directed neutrons.

Neutron measurements will be taken in Target Area III, at the locations identified by "M" in Figure 2, as soon as beam is delivered. An organic scintillator is expected to be used to identify flux densities and locations of fast fowarddirected neutrons. The Bonner Sphere will continue to be used to provide dose equivalent rate information.

The neutron measurements made in Target Area III will be needed to allow for the expected administrative controls to be fully implemented and personnel access 
allowed while beam is delivered to the area. Since all bypasses are based on a radiation measurement, the information gained is necessary for selecting one or more survey instruments that will be needed to correctly assess the radiation hazards. The information will also be used to determine the construction of beam stops required to either attenuate or moderate high energy neutrons.

\section{References}

(1) R. H. Cooke and R. A. Wynveen, IEEE Transactions on Nuclear Science, Vol. NS-30, No. 2, April 1983, pp. 1588-1591. 